\title{
Congenital Asplenia Revealed by Streptococcus oralis septicemia: Case Report
}

Mouna EL Fane*, Mustapha Sodqi, Latifa Marih, Abdelfattah Chakib and Kamal Marhoum El Filali

Department of Infectious Diseases, UHC Ibn Rochd, Casablanca, Morocco

"Corresponding author: Mouna EL Fane, Department of Infectious Diseases, UHC Ibn Rochd, Casablanca, Morocco, Tel: 212 660832362; E-mail: elfanemouna@gmail.com

Received date: May 01, 2017; Accepted date: May 16, 2017; Published date: May 23, 2017

Copyright: (C) 2017 EL Fane M, et al. This is an open-access article distributed under the terms of the Creative Commons Attribution License, which permits unrestricted use, distribution, and reproduction in any medium, provided the original author and source are credited.

\begin{abstract}
Congenital asplenia is a rare life-threatening condition, often presenting with sepsis caused by encapsulated pathogens. It may arise as part of situs abnormalities or result from an unrelated specific defect of spleen development. Isolated congenital asplenia is a very rare condition. We report a case of asplenia revealed by severe sepsis and multi-organ failure in a previously healthy 25-year-old male how had never undergone any surgical procedures. Blood cultures grew Streptococcus oralis four days after admission. Computed tomography revealed pneumonia and asplenia. The patient was finally diagnosed as Streptococcal sepsis revealing isolated congenital asplenia. Cephalosporin and levofloxacin were administered and the patient died following cardiopulmonary arrests. No family history for this condition was reported. Clinicians should pay attention to the congenital asplenia in Streptococcal disease, particularly in the event of overwhelming sepsis. In affected individuals, the use of appropriate antibiotic prophylaxis and immunisations could save lives.
\end{abstract}

Keywords: Congenital asplenia; Sepsis; Streptococcus oralis, Vaccination

\section{Introduction}

Asplenia is an uncommon condition that may be acquired following surgery, functional or congenital [1]. Congenital asplenia is often occurs in the context of a recognised malformation syndrome, the Ivemark syndrome, also called asplenia syndrome [2,3]. Conversely, isolated congenital asplenia is rare, with only 33 cases reported in the literature [1]. Different modes of inheritance have been reported for these syndromes, mostly autosomal recessive, exceptionally autosomal dominant or X-linked [4]. Most of these presented in the early years of life and only seven cases were diagnosed in the adult [1]. Commonly, the infections strictly correlated to the absence of the spleen are due to encapsulated bacteria that are the most virulent pathogens in this set of patients [5]. They can produce a serious fulminant illness that carries a high mortality rate [6]. The authors report another case of this condition in a 25 -year-old man presenting with Streptococcus oralis sepsis revealing congenital asplenia.

\section{Case Presentation}

A previously healthy 25-year-old male was admitted to emergency due to severe sepsis, and multi-organ failure in April 2017. He developed general fatigue, dyspnea and a high-grade fever on the day before hospitalization. He was not on any medications and had never undergone any surgical procedures. Physical examination revealed discolored conjunctiva, bilateral pulmonary rales. The patient had a fever $\left(39^{\circ} \mathrm{C}\right)$, hypotensive $(100 / 50 \mathrm{mmHg})$, tachycardic (pulse of 180 per minute) and olyguric. There were signs of respiratory distress with supra sternal retractions and respiratory rate was 35 breaths per minute. The patient was noted to have a lower limb pain. Chest radiograph showed a bilateral basal pulmonary opacity (Figure 1). A CT scan of the thorax and abdomen revealed the absence of the spleen, condensation foci of both lower pulmonary lobes and left pleural effusion of medium abundance (Figure 2). Laboratory studies were compatible with a systemic inflammatory response syndrome and multiple organ failure as follows: white blood cell (WBC) count 43 $200 / \mu$ l (neutrophils $80 \%$, lymphocytes $10.5 \%$ ) (reference range WBC

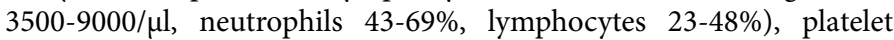
count 352,000/ $\mu \mathrm{l}$ (reference range 130,000-370,000/ $\mu \mathrm{l}$ ), hemoglobin $6.2 \mathrm{~g} / \mathrm{dl}$. Blood tests revealed markedly deranged renal function tests (serum creatinine $207 \mu \mathrm{mol} \mathrm{L} \mathrm{L}^{-1}$, urea $24 \mathrm{mmol} \mathrm{L}^{-1}$ ) and hepatic function, aspartate aminotransferase $83 \mathrm{IU} / \mathrm{L}$ (reference range 8-37 $\mathrm{IU} / \mathrm{l}$ ), alanine transaminase $50 \mathrm{IU} / \mathrm{l}$ (reference range 4-44 IU/l), total bilirubin $53 \mathrm{mg} / \mathrm{dl}$ (reference range 0.2-0.9 mg/dl). Microbiology cultures from the blood sample yielded Streptococcus oralis seven days after admission. Streptococcus oralis was sensitive to clindamycin, chlorampheicol, vancomycin and levofloxacin. It was resistant to ceftriaxone.

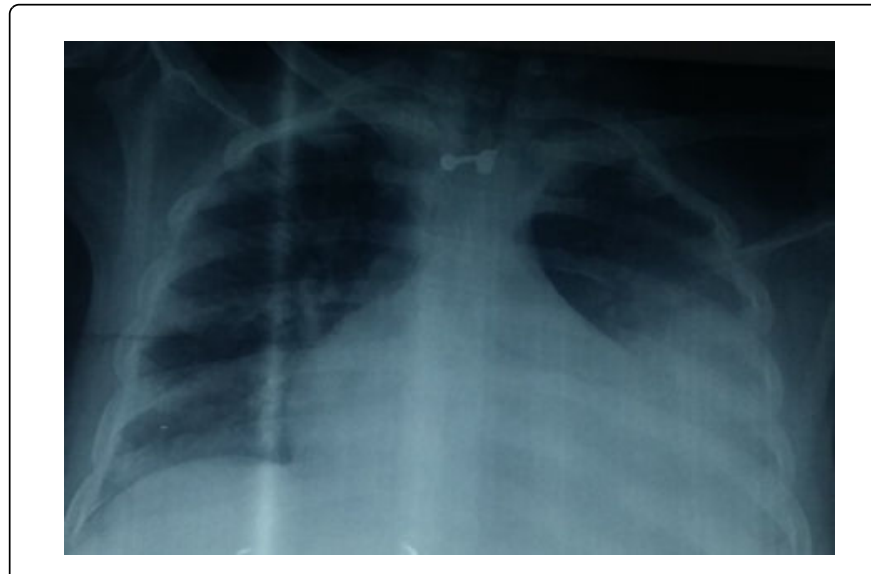

Figure 1: Chest radiograph showing a bilateral basal pulmonary opacity. 


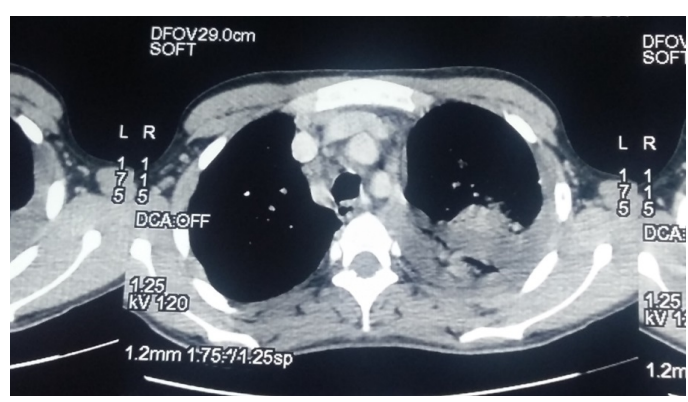

Figure 2: CT scan of the thorax revealing the condensation foci of both lower pulmonary lobes and left pleural effusion of medium abundance.

Cardiac echography was normal, he didn't show vegetation. Congenital asplenia followed by Streptococcus oralis septicemia was strongly evoked. An injectable antibiotic based on ceftriaxone (2 gram per day) and levofloxacin (500 mg per day) was administered on the day of admission of the patient. Although therapy including vasopressor drugs, blood transfusion, the patient developed multiorgan failure requiring circulatory, ventilator and renal support. The patient died following cardiopulmonary arrests.

\section{Discussion}

The patient had never splenectomized. This was his first episode of septicemia and he had never received pneumococcal vaccine. The patient quickly developed septic shock, multi-organ failure before dying. This case of streptococcal septicemia in a previously well man illustrates how infection can rapidly progress in patients who have a congenital asplenia. Congenital asplenia is most often found in association with other anomalies [1]. The most common of these anomalies is Ivemark syndrome. This is characterized by visceral heterotaxy with bilateral right-sidedness. The right-sided organs are duplicated and organs that are normally present on the left side are absent [7]. Congenital isolated asplenia is a separate entity due to a specific defect of spleen development [4]. He is rare and only 33 cases have been reported in the literature. Of these, 20 (54\%) were shown to be familial and $13(46 \%)$ sporadic like our case [8]. Clinical presentation of congenital isolated asplenia is heterogeneous. It can be represented by pneumococcal sepsis, meningitis, sepsis of unknown origin, thrombocytosis [9]. Asplenia is also a risk factor for overwhelming sepsis $[10,11]$. The clinical characteristics of our patient were compatible with the summary. Congenital asplenia is also appears to be heterogeneous in its inheritance as, although the majority of families reported show autosomal dominant inheritance, there are at least two families in the literature now which demonstrate autosomal recessive inheritance [4]. Conversely of our patient, only seven patients reported in the literature were older than 20 years of age [1]. Streptococcus pneumoniae is responsible for up to $80 \%$ of asplenia followed by sepsis cases. However, in the years, the bacterial pattern of splenectomy sepsis has been changing, Haemophilus influenza and Neisseria meningitidis are also significant $[1,12]$. Gram-negative bacilli including Salmonella species, Klebsiella species and Pseudomonas aeruginosa are less common causes of bacteraemia [1]. In the case reported, Streptococcus oralis was the cause of sepsis. Streptococcus oralis is the most virulent of the viridans streptococci [13]. It has been traditionally regarded as minor opportunist pathogens, being primarily associated with dental caries [1]. However, over the past decade these bacteria have increasingly recognized as causes of often fatal septicaemias in immune-compromised patients $[10,13]$. The mechanisms by which Streptococcus oralis causes this wide range of infections are as yet unclear, but the sialidase produced by this bacterium has been proposed as contributing towards the pathogenicity of several other micro-organisms, including Streptococcus pneumonia [13]. Streptococcus oralis colonize tooth surfaces as well as mucosal membranes. Because of their presence in the bacterial deposits on tooth surfaces, these species may enter the bloodstream during dental procedures. In healthy individuals, such bacteria of low virulence are cleared from the circulation within one hour. However, in patients with predisposing conditions, the circulating streptococci may settle in a niche protected from phagocytic cells [10]. Blood cultures are of value in patients with invasive streptococcal infections. This is also the case in patients with suspected pneumonia, particularly when this is severe, since up to $15 \%$ of patients will be bacteraemic [10]. The diagnosis of asplenia can also be confirmed by identification of Howell-Jolly bodies in the erythrocyte membrane using phase contrast microscopy and the absence of the spleen noted on ultrasound examination of the abdomen [4]. In a review of the literature, cases of congenital asplenia were referred to hospitals within several hours to two days from the time of onset. However, these patients can deteriorate very rapidly, with death occurring within several days or even hours. This accelerated course of the disease is related to reduced response to antigen, impaired phagocytosis and decreased levels of tuftsin and properdin $[1,11]$. Mortality after acute presentation with sepsis is $56 \%$ $[1,14,15]$. Other series have reported up to an $80 \%$ mortality arising from sepsis $[16,17]$.

For prevention of overwhelming sepsis, guidelines concerning the management of asplenic patients, recommends vaccination (pneumococcal and Hib vaccines), antibiotic prophylaxis with penicillin $[4,18,19]$. However, in the case of congenital isolated asplenia, sepsis may be the first sign of the disease [4]. Vaccine administration might have changed the clinical outcome of our patient. The duration of antibiotic prophylaxis being unclear, adherence to taking daily medications, and the acquisition of drugresistant bacteria are current unresolved problems [20]. Asplenia detected by CT or ultrasonography by chance, present opportunities to consider preventive measures and warn the patient about the risk of overwhelming sepsis [18].

\section{Conclusion}

We have reported a case of overwhelming streptococcal sepsis with asplenia as evidenced by CT scan evaluation. Through this case and a review of the literature, we consider that asplenia is a potential risk factor for overwhelming streptococcal sepsis. Clinicians should carefully check splenic size in patients with sepsis of unknown origin.

\section{References}

1. Thiruppathy K, Privitera A, Jain K, Gupta S (2008) Congenital asplenia and group B Streptococcus sepsis in the adult: Case report and review of the literature. FEMS Immunol Med Microbiol 53: 437-439.

2. Freedom RM (1972) The asplenia syndrome: A review of significant extracardiac structural abnormalities in 29 necropsied patients. J Pediatr 81: $1130-1133$. 
Citation: EL Fane M, Sodqi M, Marih L, Chakib A, El Filali KM (2017) Congenital Asplenia Revealed by Streptococcus oralis septicemia: Case Report. J Antimicrob Agents 3: 142. doi:10.4172/2472-1212.1000142

Page 3 of 3

3. Ivemark BI (1955) Implications of agenesis of the spleen on the pathogenesis of cono-truncus anomalies in childhood. Acta Paediatr Scand 44: 1-11.

4. Ahmed SA, Zengeya S, Kini U, Pollard AJ (2010) Familial isolated congenital asplenia: Case report and literature review. Eur J Pediatr 169 315-318.

5. Edgren G, Almqvist R, Hartman M, Utter G (2014) Splenectomy and the risk of sepsis: A population-based cohort study. Ann Surg 260 1081-1087.

6. Ejstrud P, Kristensen B, Hansen JB, Madsen KM, Schønheyder HC, et al (2000) Risk and patterns of bacteraemia after splenectomy: A populationbased study. Sc and J Infect Dis 32: 521-525.

7. Dyke MP, Martin RP, Berry PJ (1991) Septicaemia and adrenal haemorrhage in congenital asplenia. Arch Dis Child 66: 636-637.

8. Myerson RM, Koelle WA (1956) Congenital absence of the spleen in an adult: Report of a case associated with recurrent WaterhouseFriderichsen syndrome. N Engl J Med 254: 1131-1132.

9. Zasshi G, Yahagi Y, Fujikawa H, Tsutsumi N, Inami M, et al (2013) Case report: An autopsy case of fulminent pneumococcal infection, meningitis and multiple organ failure resulting from splenic hypoplasia. Nihon Naika Gakkai Zasshi 102: 433-436.

10. Kilian M (2012) Streptococcus and enterococcus. Bacterial pathogens and associated diseases 16: 174-188.

11. Waghorn DJ (2001) Overwhelming infection in asplenic patients: Current best practice preventive measures are not being followed. J Clin Pathol 54: 214-218.
12. Gand L, Pizzigallo E (2015) Bacterial infections following splenectomy for malignant and nonmalignant hematologic diseases. Mediterr J Hematol Infect Dis 7: e2015057.

13. Byers HL, Tarelli E, Homer KA, Beighton D (2000) Isolation and characterisation of sialidase from a strain of Streptococcus oralis. J Med Microbiol 49: 235-244.

14. Halbertsma FJ, Neeleman C, Weemaes CM, Van Deuren M (2005) The absent and vanishing spleen: Congenital asplenia and hyposplenism-two case reports. Acta Paediatr 94: 369-371.

15. Hummler HD, Pohlandt F, Essig A (2005) Fulminant pneumococcal sepsis in a 13 months old child with congenital asplenia. Klin Padiatr 217: 274-275.

16. Beytout J, Tournilhac O, Laurichesse H (2003) Asplenia and hyposplenism. Presse Med 32: 5-9.

17. Dyke MP, Martin RP, Berry PJ (1991) Septicaemia and adrenal haemorrhage in congenital asplenia. Arch Dis Child 66: 636-637.

18. Yaita K, Sakamoto Y, Nakaharai K, Yoshimura Y, Tachikawa N (2014) Overwhelming pneumococcal sepsis in a patient with splenic hypoplasia. General Medicine 15: 68-71.

19. Spelman D, Buttery J, Daley A, Isaacs D, Jennens I, et al. (2008) Guidelines for the prevention of sepsis in asplenic and hyposplenic patients. Intern Med J 38: 349-356.

20. Price VE, Blanchette VS, Ford-Jones EL (2007) The prevention and management of infections in children with asplenia or hyposplenia. Infect Dis Clin North Am 21: 697-710. 